\title{
ON ICA OF COMPLEX-VALUED FMRI: ADVANTAGES AND ORDER SELECTION
}

\author{
Wei Xiong, Yi-Ou Li, Hualiang Li, Tülay Adalı \\ University of Maryland, Baltimore County, \\ Department of CSEE \\ Baltimore, MD 21250
}

\author{
Vince D. Calhoun
}

\begin{abstract}
Functional magnetic resonance imaging (fMRI) data are originally acquired as complex-valued images, while virtually all fMRI studies only use the magnitude of the data in the analysis. Since little is known for devising models for the phase, independent component analysis (ICA) emerges as a promising technique for data-driven analysis of fMRI data in its native complex form. In this paper, we compare the performance of ICA on real-valued and complex-valued fMRI data and show the advantages of the complex approach. We also develop complex-valued order selection scheme to improve the estimation of the number of independent components in complex-valued fMRI data using information-theoretic criteria. Comparisons on order selection using real-valued and complex-valued fMRI data demonstrate the more informative nature of complex data.
\end{abstract}

Index Terms - fMRI, ICA, Order Selection, Complex Analysis

\section{INTRODUCTION}

FMRI is a non-invasive, powerful brain imaging technique that has been utilized since the early 1990s [1], and has provided valuable insights to the understanding of the human brain function. Even though the fMRI data is acquired as complex-valued spatial-temporal data, commonly, only the magnitude images are analyzed, and the phase of fMRI data is discarded. The complex-valued data are expected to be more informative, which implies that direct analysis on the complex-valued fMRI data may produce more useful results, provided that effective analysis tools can be adopted.

Because fMRI relies on the detection of small intensity changes over time, it poses significant challenges for data analysis techniques. Model-based analysis approaches, such as linear regression, are widely used. Generalized likelihood ratio tests have been developed for complex-valued fMRI data [2]. Model-based approaches are robust, yet often too rigid to capture the richness of the human brain activation.

ICA, on the other hand, is a data-driven approach that provides a more flexible framework for the analysis of fMRI data [3]. The advantage of ICA lies in its ability to estimate cognitive processes when detailed a priori models are not available.

This work is supported by the NSF grants NSF-CCF 0635129 and NSFIIS 0612076.
ICA of complex-valued fMRI has been noted as a promising method for the study of fMRI data [4], [5]. High dimensionality and high noise level of the fMRI data suggest that order selection is critical to the success of the ICA approach, because overestimation or underestimation of the ICA model causes problems in the reliability of the estimation. In [6], the order estimation problem is addressed for real-valued fMRI data and a subsampling scheme to improve order selection performance has been proposed. In this paper, we extend the work to improve order selection performance for complex-valued fMRI data. We make comparisons between real-valued and complex-valued fMRI data using ICA and show the advantage of using the complex approach.

In the next section, we discuss fMRI and introduce an ICA approach in the complex domain. In section 3, we develop an order selection scheme in complex domain based on independent, identically distributed (i.i.d.) samples. Furthermore, we show experimental results on order selection and ICA estimation, both on simulated and actual fMRI data. We conclude the paper with a discussion of the results.

\section{COMPLEX-VALUED ICA OF FMRI}

The MRI signal is acquired as a quadrature signal using two orthogonal detectors, to compose a complex form in frequency domain ( $k$-space). Then through inverse Fourier transform, the complex-valued fMRI data in the spatial domain is obtained, and typically the magnitude of the data is used in further analysis. Although the phase of complex fMRI data may be more prone to noise effects, recent work has shown the potential of using the complex-valued data in the analysis [7].

We assume independence of spatial brain activations (spatial ICA) of fMRI data, and write the complex ICA model as:

$$
\mathbf{X}=\sum_{k=1}^{M} \mathbf{a}_{k} \mathbf{s}_{k}^{T}+\mathbf{n}
$$

where $\mathbf{s}_{k}$ is the $N \times 1$ vector, which represents the activation intensity of each voxel, for the $k$ th independent spatial map; $\mathbf{a}_{k}$ is an $N \times 1$ vector, representing time course of the $k$ th independent spatial map component. Here all variables are complex-valued and $M$ is the number of informative spatial map sources, $N$ the number of voxels in each spatial map source, $T$ the number of time points in the time course, and $\mathbf{n}$ is the $T \times N$ matrix of Gaussian noise components. 
The information maximization (nonlinear decorrelation) algorithm, which is equivalent to maximum likelihood (ML), is an attractive approach for ICA estimation and it yields reliable result in real-valued fMRI analysis [8]. The complexvalued nonlinear decorrelation algorithm [4], [9] is employed in our experiments.

\section{COMPLEX-VALUED ORDER SELECTION}

\subsection{Information-theoretic criteria}

Information-theoretic criteria (ITC) are commonly used for order selection in many signal processing problems, including ICA. There are a number of ITC commonly used for order selection, such as, Akaike's information criterion (AIC) [10], Kullback-Leibler information criterion (KIC) [11], and the minimum description length criterion (MDL) [12]. The formulas for AIC, KIC and MDL criteria have similar structures [13]:

$$
\begin{gathered}
\mathcal{E}_{A I C}(k)=-2 \mathcal{L}\left(\mathbf{x} \mid \boldsymbol{\Theta}_{k}\right)+2 \mathcal{G}\left(\boldsymbol{\Theta}_{k}\right) \\
\mathcal{E}_{K I C}(k)=-2 \mathcal{L}\left(\mathbf{x} \mid \boldsymbol{\Theta}_{k}\right)+3 \mathcal{G}\left(\boldsymbol{\Theta}_{k}\right) \\
\mathcal{E}_{M D L}(k)=-\mathcal{L}\left(\mathbf{x} \mid \boldsymbol{\Theta}_{k}\right)+\frac{1}{2} \mathcal{G}\left(\boldsymbol{\Theta}_{k}\right) \log N
\end{gathered}
$$

where $\mathcal{L}\left(\mathbf{x} \mid \Theta_{k}\right)$ is the maximum log-likelihood of the observations $\mathbf{x}$, based on the model parameter set $\boldsymbol{\Theta}_{k}$ and $\mathcal{G}\left(\boldsymbol{\Theta}_{k}\right)$ the penalty for model complexity given by the total number of free parameters in $\Theta_{k}$. For MDL, the penalty term is scaled by $\log N$ where $N$ is the sample size.

The maximum log-likelihood for complex-valued data is given by

$$
\mathcal{L}\left(\mathbf{x} \mid \Theta_{k}\right)=N \log \left(\frac{\prod_{i=k+1}^{T} \lambda_{i}^{1 /(T-k)}}{\frac{1}{T-k} \sum_{i=k+1}^{T} \lambda_{i}}\right)^{T-k}
$$

where $T$ is the original dimension of the multivariate data, $k$ is the candidate order, $N$ is the sample size, and $\lambda_{i}$ 's are the eigenvalues of the sample covariance matrix of the multivariate observations. The number of free parameters in $\mathcal{G}\left(\boldsymbol{\Theta}_{k}\right)$ for complex-valued data is given by

$$
\mathcal{G}\left(\boldsymbol{\Theta}_{k}\right)=1+2 T k-k^{2} .
$$

\subsection{IID sampling in complex domain}

Order selection formulations are typically based on an i.i.d. sample assumption [13]. However, there is inherent spatial smoothness in the fMRI data due to the point spread function of the scanner. Furthermore, smoothing is a common preprocessing step used to suppress the high frequency noise in the fMRI data. Both factors contribute to dependence among the samples in fMRI volume data, which leads to over-estimation of the order number. To address this problem, a subsampling scheme is proposed to identify an effectively i.i.d. sample set from the whole fMRI volume for real-valued data [6]. The method can be extended to the complex case as we discuss next.
Given a complex-valued Gaussian random process $z[n]=$ $x[n]+i y[n], n=1,2, \ldots, N$, where the real and imaginary parts are assumed to be uncorrelated, the entropy rate of complex-valued Gaussian process $z[n]$ is the sum of the entropy rate of the real part $x[n]$ and imaginary part $y[n]$, expressed as

$h_{z}=h_{x}+h_{y}=\ln (2 \pi e)+\frac{1}{4 \pi} \int_{-\pi}^{\pi} \ln s_{x}(\omega) d \omega+\frac{1}{4 \pi} \int_{-\pi}^{\pi} \ln s_{y}(\omega) d \omega$

where $s(\omega)$ is the power spectral density function. For the fMRI data used in this study, the correlation values for Gaussian sequences obtained through principal component analysis (PCA) are $0.058 \pm 0.026$. Thus the uncorrelatedness condition is approximately satisfied. In [14], it is shown that the real and imaginary parts of MR images can be characterized as independent random variables.

For a complex-valued stationary Gaussian random process, if the real and imaginary parts are independent, the upper bound of the entropy rate is $\ln (2 \pi e)$ and the upper bound is achieved if and only if all samples of the process are i.i.d. By comparing the entropy rate of the subsampled data with that of an i.i.d. process, the grid of locations on which the data samples are considered to be effectively independent is determined. Therefore, an effective i.i.d. sample set is obtained on this grid of spatial locations at which the dependence among the samples is small enough to be ignored. Since the subsampling procedure decreases the number of samples for estimation, an eigenspectrum adjustment scheme [6] is used to mitigate the finite sample effect.

\section{EXPERIMENT}

\subsection{Order selection on simulated data}

We generate eight complex-valued spatial maps to simulate the fMRI sources and corresponding time courses, the magnitudes of which are similar to the ones used in [8]. In the fMRI experiment, the phase difference induced by the task activation is typically less than $\pi / 9$ [7], [15]. Therefore, we keep the phase of each pixel uniformly distributed in the range $[-\pi / 18, \pi / 18]$. The phase of each complex-valued time point is generated proportional to its magnitude, but restricted to a small range, which is less than $\pi / 18$. The spatial sources are rearranged into one-dimensional vectors and mixed by the corresponding time courses, as in Eq. (1). Complexvalued Gaussian noise is added to the data set with a specified contrast to noise ratio (CNR). The mixture data are spatially smoothed, separately for the real and imaginary parts, by a Gaussian kernel with the full-width at half maximum (FWHM) of 2 pixels.

The complex-valued mixtures of eight sources, with different noise levels of $\mathrm{CNR}=-3,0,3$ and $6 \mathrm{~dB}$, are created and the complex-valued order selection described in Section 3.2 is applied to these mixtures. The criteria used in the experiment are AIC, KIC and MDL. Fig. 1 shows the result of 10 Monte Carlo simulations where a different noise realization is used for each run. The standard deviation is stacked on the mean value in each bar plot.

As shown in Fig. 1, the criteria yield accurate estimates when the CNR is higher than $0 \mathrm{~dB}$. Without subsampling, the 


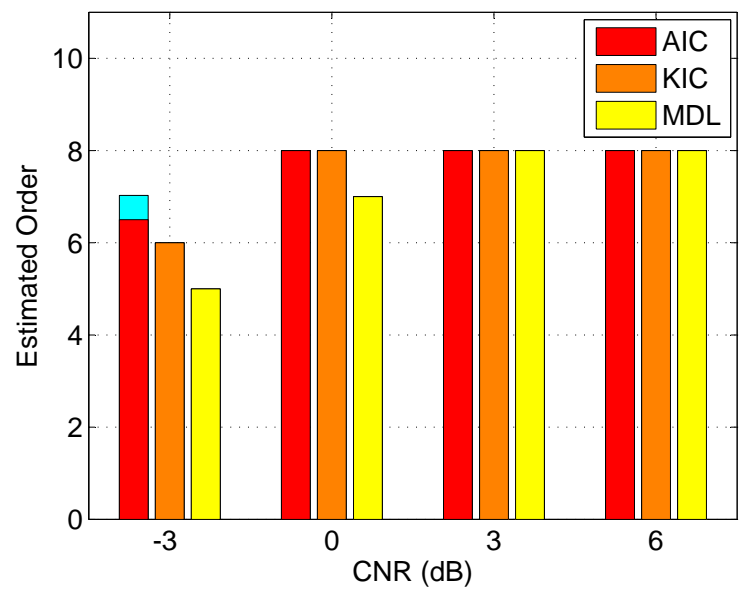

Fig. 1. Order selection on simulated data with different CNR values: $-3,0,3$ and $6 \mathrm{~dB}$

order number is significantly overestimated, e.g., $98 \pm 1$ by $\mathrm{AIC}, 95 \pm 2$ by KIC and $68 \pm 6$ by MDL for CNR $=3 \mathrm{~dB}$. In another experiment, we randomly choose four sources from all eight sources and mix them together at different CNR values. The true order is estimated when the CNR value is higher than $0 \mathrm{~dB}$. CNR of actual fMRI data is typically in the range $0-3 \mathrm{~dB}$, and our complex-valued order selection scheme is effective for this CNR range.

\subsection{FMRI data}

Eight sets of fMRI data, which are related to a visuomotor task, are the same data used in [6]. The MATLAB Toolbox for Statistical Parametric Mapping (SPM) is used for motion correction to magnitude data and the resulting parameters are applied to real and imaginary part of the complex-valued fMRI data, resulting in the motion-corrected complex images. Then the real and imaginary images are spatially smoothed with an $8 \times 8 \times 8 \mathrm{~mm}$ FWHM Gaussian kernel, to improve CNR of complex-valued fMRI data. The magnitude of smoothed complex-valued data is taken as real-valued fMRI data.

A real-valued order selection scheme in [6] and a complexvalued scheme described in Section 3 are applied to the fMRI data. The criteria used in the experiment are AIC, KIC and MDL. Fig. 2 shows the result based on eight subjects. The standard deviation across different subjects is stacked on the mean value in each bar plot. It is observed that the order estimated for complex-valued fMRI data is higher than that of real-valued data, which indicates that complex-valued fMRI data contain more information than real-valued data. For subsequent ICA results, we use the order estimated by AIC, i.e., 20 for real-valued data and 30 for complex-valued data.

Using PCA, the real-valued data are reduced from 119 time points to 20 principal components and the complex-valued data to 30. An ICA algorithm, using nonlinear decorrelations with the nonlinear function $\operatorname{atanh}(\cdot)$, is applied to the dimension reduced data. The time-courses and spatial maps are reconstructed after ICA. For the real-valued fMRI data,

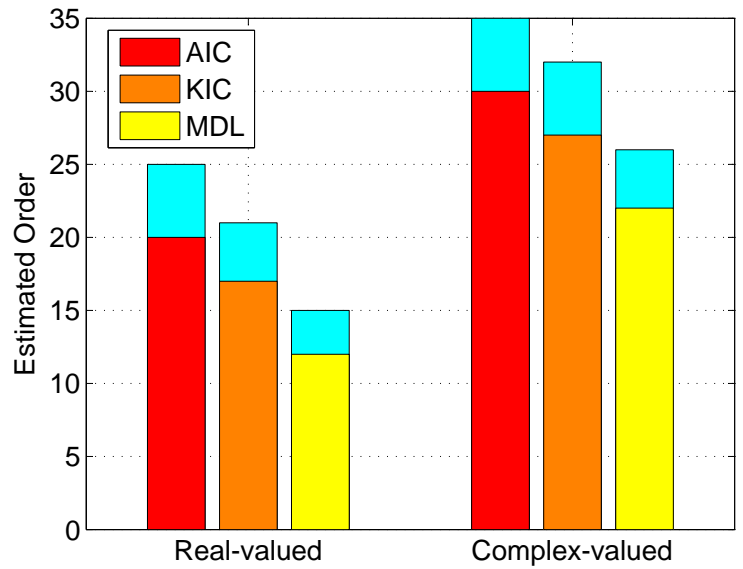

Fig. 2. Order selection on real-valued and complex-valued fMRI data

the resulting spatial maps and for the complex-valued fMRI data, the magnitudes of complex spatial maps are converted to $Z$-scores. All maps are thresholded at $|Z|>2$.

In our experiments, the voxels of whole brain images are applied in ICA estimation, which improves statistical power compared with previous work that restricts ICA analysis to the posterior half of the brain [5], [15]. For performance comparison, three typical spatial components associated with the task are used, left task-related (LTR), right task-related (RTR) and the default mode (DM). Fig. 3 shows the spatial activation map of DM from real-valued and complex-valued data in one experiment. We perform ICA estimations 10 times with random initial conditions for each subjects and calculate the average. Fig. 4 shows the comparison of number of voxels in the thresholded activation area and Fig. 5 shows the comparison of maximum and mean of $Z$-scores in the activation area. The standard deviation across different subjects is stacked on the mean value in each bar plot.

For all three components in Fig. 4, number of voxels in activation area from complex-valued fMRI data is larger than that from real-valued data. Fig. 5 shows that the maximum and mean of $Z$-scores in activation area from complexvalued fMRI data are higher than that from real-valued data. We show an example of DM from one experiment in Fig. 3. The increase of number of voxels, maximum and mean of $Z$ scores indicates higher sensitivity of the estimation method in complex-valued approach.

\section{DISCUSSION}

Through comparisons of the performance of ICA on realvalued and complex-valued fMRI data, we observe that complex ICA applied to fMRI data increases the sensitivity of ICA for estimating brain activations. Optimization of complexvalued ICA algorithms need to be studied in more detail to facilitate fMRI analysis. We develop an improvement for order selection in complex domain to address overestimation of the order due to voxel dependence. The implementation to ac- 




(a)

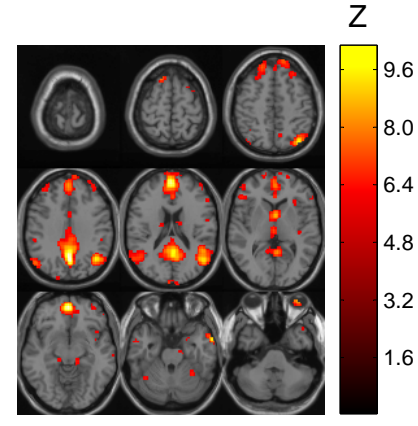

(b)
Fig. 3. Spatial activation map of DM, (a) from real-valued data, (b) from complex-valued data

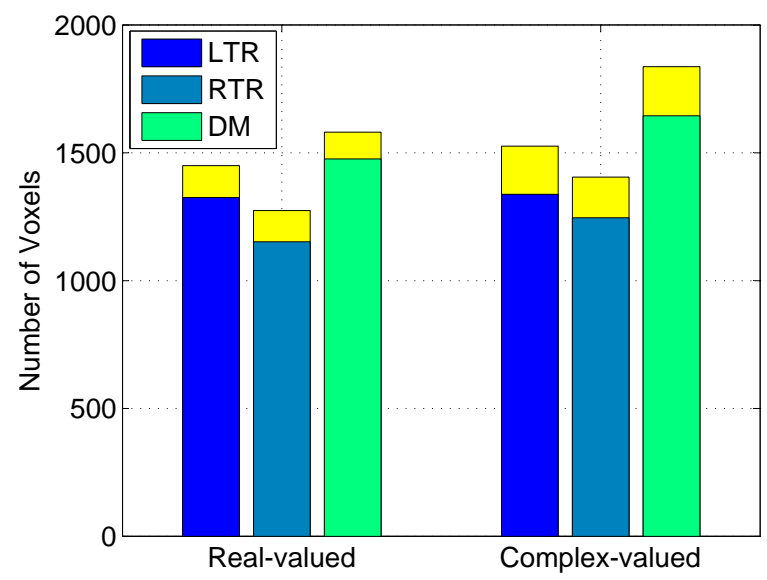

Fig. 4. Number of voxels in activation area of components estimated from real-valued and complex-valued fMRI data, $Z>2$

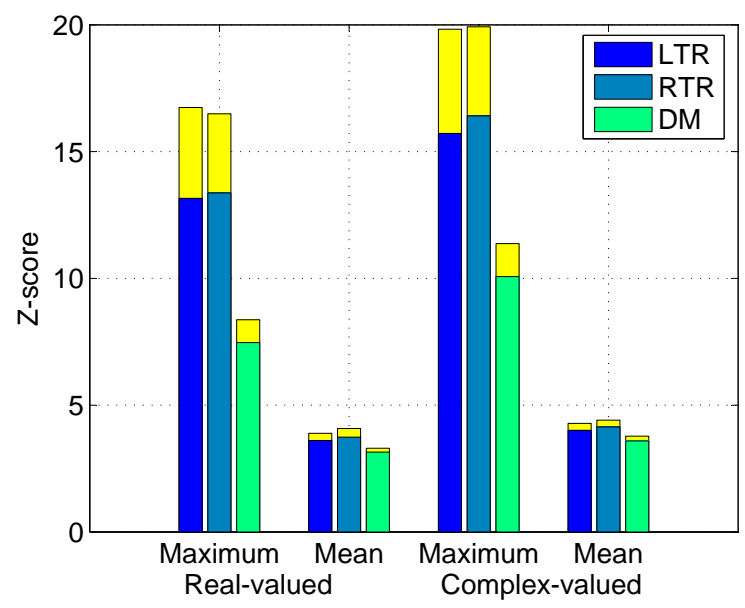

Fig. 5. Maximum and mean of $Z$-scores in activation area of components estimated from real-valued and complex-valued fMRI data, $Z>2$ tual fMRI data demonstrates that the order of complex-valued fMRI data is higher than that of real-valued data, which suggests that complex-valued fMRI data might be more informative than real-valued data. The effect of order selection on the performance of complex ICA algorithms deserves further investigation.

\section{REFERENCES}

[1] S. Ogawa, S.D.W. Tank, R. Menon, J.M. Ellermann, S.G. Kim, H. Merkle, and K. Ugurbil, "Intrinsic signal changes accompanying sensory stimulation: functional brain mapping with magnetic resonance imaging," Proc. Nat. Acad. Sci., vol. 89, pp. 5851-5955, 1992.

[2] F. Y. Nan and R. D. Nowak, "Generalized likelihood ratio detection for fMRI using complex data," IEEE Trans. Med. Imaging, vol. 18, pp. 320-329, 1999.

[3] M. J. McKeown, S. Makeig, G. G. Brown, T.-P. Jung, S. S. Kindermann, A. J. Bell, and T. J. Sejnowski, "Analysis of fMRI data by blind separation into independent components," Hum. Brain Mapping, vol. 6, pp. 160-188, 1998.

[4] T. Adal1, T. Kim, and V. Calhoun, "Independent component analysis by complex nonlinearities," in Proc. ICASSP, Montreal, Canada, 2004, vol. 5, pp. 525-528.

[5] V. D. Calhoun, T. Adal1, and Y.-O. Li, "Independent component analysis of complex-valued functional MRI data by complex nonlinearities," in Proc. ISBI, Arlington, VA, 2004.

[6] Y.-O. Li, T. Adalı, and V. D. Calhoun, "Estimating the number of independent components for fMRI data," Hum. Brain Mapping, in press.

[7] F. G. Hoogenrad, J. Reichenbach, E. Haacke, S. Lai, K. Kuppusamy, and M. Sprenger, "In vivo measurement of changes in venous blood-oxygenation with high resolution functional MRI at .95 Tesla by measuring changes in susceptipility and velocity," Mag. Res. Med., vol. 39, pp. 97-107, 1998.

[8] N. Correa, Y.-O. Li, T. Adal1, and V. D. Calhoun, "Comparison of blind source separation algorithms for fMRI using a new Matlab toolbox: GIFT," in Proc. ICASSP, Philadelphia, PA, 2005, vol. 5, pp. 401-404.

[9] T. Adalı and H. Li, "A practical formulation for computation of complex gradients and its application to maximum likelihood," in Proc. ICASSP, Honolulu, Hawaii, 2007.

[10] H. Akaike, "A new look at statistical model identification," IEEE Trans. Auto. Cont., vol. 19, pp. 716-723, 1974.

[11] J. E. Cavanaugh, "A large-sample model selection criterion based on Kullback's symmetric devergence," Stat. and Prob. Letters, vol. 44, pp. 333-344, 1999.

[12] J. Rissanen, "Modeling by the shortest data description," $A u$ tomatica, vol. 14, pp. 465-471, 1978.

[13] M. Wax and T. Kailath, "Detection of signals by information theoretic criteria," IEEE Trans. Acoust., Speech, and Signal Processing, vol. 33, pp. 387-392, 1985.

[14] T. Lei and F. W. Wehrli, "Magnetic resonance (MR) image analysis - a statistical approach," Int. Journal of Image and Graphics, vol. 7, pp. 119-141, 2007.

[15] V. D. Calhoun, T. Adalı, P. C. M. van Zijl, and J. J. Pekar, "Independent component analysis of fMRI data in the complex domain," Mag. Res. Med., vol. 48, pp. 180-192, 2002. 